\title{
Intensity of statin therapy and renal outcome in chronic kidney disease: Results from the Korean Cohort Study for Outcome in Patients With Chronic Kidney Disease
}

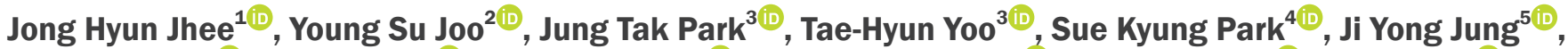

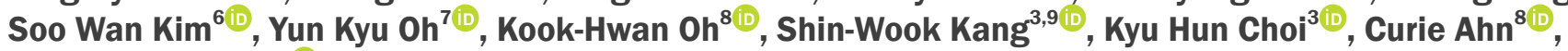 \\ Seung Hyeok $\mathrm{Han}^{3(1 D}$ \\ 'Division of Nephrology, Department of Internal Medicine, Gangnam Severance Hospital, Yonsei University College of Medicine, Seoul, \\ Republic of Korea \\ 'Division of Nephrology, Department of Internal Medicine, Myongji Hospital, Goyang, Republic of Korea \\ ${ }^{3}$ Department of Internal Medicine, College of Medicine, Institute of Kidney Disease Research, Yonsei University, Seoul, Republic of Korea \\ ${ }^{4}$ Department of Preventive Medicine, Seoul National University College of Medicine, Seoul, Republic of Korea \\ ${ }^{5}$ Division of Nephrology, Department of Internal Medicine, Gachon University Gil Medical Center, Incheon, Republic of Korea \\ ${ }^{6}$ Department of Internal Medicine, Chonnam National University Medical School, Gwangju, Republic of Korea \\ ${ }^{7}$ Department of Internal Medicine, SMG-SNU Boramae Medical Center, Seoul, Republic of Korea \\ ${ }^{8}$ Department of Internal Medicine, Seoul National University Hospital, Seoul, Republic of Korea \\ ${ }^{9}$ Department of Internal Medicine, College of Medicine, Severance Biomedical Science Institute, Brain Korea 21 PLUS, Yonsei University, \\ Seoul, Republic of Korea
}

\begin{abstract}
Background: Higher statin intensity is associated with a lower risk of mortality in patients with cardiovascular disease. However, little is known about the relationship between statin intensity and chronic kidney disease (CKD) progression. Methods: We studied whether statin intensity affects kidney function decline in 1,073 patients from the Korean Cohort Study for Outcome in Patients With Chronic Kidney Disease. The participants were classified based on statin intensity as low, moderate, and high. The study endpoint was CKD progression (composite of doubling of serum creatinine, $\geq 50 \%$ decrease in estimated glomerular filtration rate [eGFR] from baseline, or end-stage renal disease). Results: The mean age was $56.0 \pm 11.4$ years, and 665 (62.0\%) participants were male. The mean eGFR was $51.7 \pm$ $26.7 \mathrm{~mL} / \mathrm{min} / 1.73 \mathrm{~m}^{2}$; there were no differences in baseline eGFR among statin intensity groups. During the median follow-up of 39.9 (25.4-61.6) months, 255 (23.8\%) patients reached the study endpoint. In multivariable Cox model after adjustment of confounders, the hazard ratios (95\% confidence interval) for adverse kidney outcome were 0.97 $(0.72-1.30)$ and $1.15(0.60-2.20)$ in moderate and high statin intensity groups, respectively, compared with the low intensity group. In addition, no significant association was observed in subgroups stratified by age, sex, eGFR, and atherosclerotic cardiovascular disease risk scores.

Conclusion: We did not observe any significant association between intensity of statin therapy and progression of CKD. Long-term kidney outcomes may not be affected by statin intensity.
\end{abstract}

Keywords: Chronic kidney disease, Disease progression, Intensity, Statin

Received January 14, 2020; Revised February 13, 2020; Accepted February 13, 2020

Edited by Dong-Ryeol Ryu, Ewha Womans University, Seoul, Republic of Korea

Correspondence: Seung Hyeok Han

Department of Internal Medicine, College of Medicine, Institute of Kidney Disease Research, Yonsei University, 50-1 Yonsei-ro, Seodaemun-gu, Seoul 03722, Republic of Korea. E-mail: hansh@yuhs.ac

Copyright (C) 2020 by The Korean Society of Nephrology

(a) This is an open-access article distributed under the terms of the Creative Commons Attribution Non-Commercial License (http://creativecommons.org/ licenses/by-nc-nd/4.0/), which permits unrestricted non-commercial use, distribution, and reproduction in any medium, provided the original work is properly cited. 


\section{Introduction}

Chronic kidney disease (CKD) is a major health problem worldwide $[1,2]$. Patients with CKD have increased risk of end-stage renal disease (ESRD), cardiovascular disease, and all-cause mortality [2-4]. Risk factors for development and progression of CKD are similar to those implicated in cardiovascular disease, including hypertension, diabetes, and dyslipidemia [5-7]. Among these factors, abnormal lipid metabolism is common in patients with kidney disease [8]. Previous experimental studies have shown that hyperlipidemia is associated with kidney injury $[9,10]$. Several epidemiologic studies demonstrated that dyslipidemia is significantly associated with increased risk of reduced kidney function or greater decline in estimated glomerular filtration rate (eGFR) in the general population without kidney disease $[11,12]$. However, the effects of statins on kidney disease progression in patients with CKD remain controversial [13].

High-intensity statin therapy can reduce the risk for cardiovascular events regardless of baseline risk $[14,15]$. The 2013 American College of Cardiology/American Heart Association (ACC/AHA) lipid guidelines recommend statin dosing in adults based on intensity, rather than targeting specific low-density lipoprotein cholesterol (LDL-C) concentration. The 2013 Kidney Disease: Improving Global Outcomes (KDIGO) lipid guidelines also recommend use of statins for most patients with CKD aged $\geq 50$ years and with eGFR $<60 \mathrm{~mL} / \mathrm{min} / 1.73 \mathrm{~m}^{2}$, in light of their high cardiovascular risk, based on previous randomized trial data on lipid-lowering therapy in CKD $[16,17]$. However, there have been conflicting results regarding the beneficial effect of statins on kidney function. Moreover, observational studies have reported an increased risk of acute kidney injury after initiation of high-intensity statin $[18,19]$, raising concern for worsening kidney function in patients with CKD and long-term use of high-intensity statin. It is well-known that statin therapy in patients with CKD is not as effective as in people without CKD. The reason for this is not clear, but we hypothesized that statin intensity might modify the relationship between statin use and adverse clinical outcomes in patients with CKD.

Thus, we aimed to investigate whether higher-intensity statin therapy is associated with lower risk of CKD progression in Korean adults with CKD.

\section{Methods}

\section{Study participants}

The Korean Cohort Study for Outcome in Patients With Chronic Kidney Disease (KNOW-CKD) is a prospective nationwide cohort study investigating various clinical courses and risk factors for CKD progression in Korean patients. Patients aged between 20 and 75 years with CKD stages from 1 to 5 before dialysis who voluntarily provided informed consent were enrolled from nine tertiary-care general hospitals throughout Korea between June 2011 and February 2016. The study rationale, design, methods, and protocol summary are provided in detail elsewhere (NCT01630486 at http://www.clinicaltrials.gov) [20]. Among 2,238 patients in the KNOW-CKD cohort, 1,085 with baseline eGFR $<15 \mathrm{~mL} / \mathrm{min} / 1.73 \mathrm{~m}^{2}$ and missing data regarding statin therapy or lipid profiles were excluded. Finally, 1,073 patients were included in the present analysis (Fig. 1). All included patients provided written informed consent, and the study was approved by the institutional review board of each participating center. All investigators conducted this study in accordance with the guidelines of the 2008 Declaration of Helsinki.

\section{Data collection}

Demographic data were collected from all patients, including age, sex, smoking status, social history, and medical history. Smoking status was classified as never, past, or current. Education level was categorized as low, less than

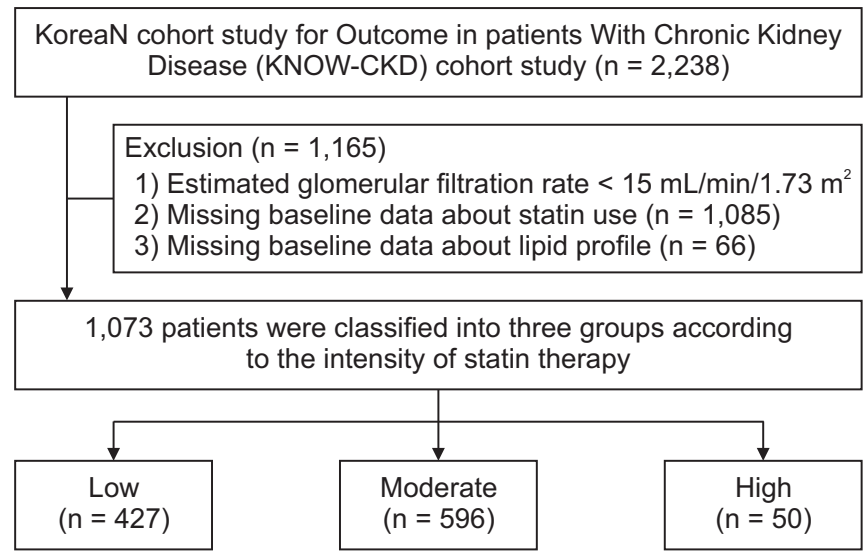

Figure 1. Study participants. CKD, chronic kidney disease. 
middle school; middle, middle school; and high, more than middle school. Income level was divided into three groups: low, $<\$ 1,200$ per month; middle, $\$ 1,200$ to $\$ 3,800$ per month; and high, $\geq \$ 3,800$ per month. Anthropometric data including height and weight were collected upon enrollment. Body mass index (BMI) was calculated by dividing initial body weight by height in meters squared. After 5 minutes of seated rest, blood pressure (BP) was measured using an electronic sphygmomanometer. Hypertension was defined as history of physician-diagnosed hypertension, systolic BP (SBP) $\geq 140 \mathrm{mmHg}$, diastolic BP $\geq$ $90 \mathrm{mmHg}$, or use of antihypertensive drugs. Diabetes was defined as history of physician-diagnosed diabetes, fasting glucose $\geq 126 \mathrm{mg} / \mathrm{dL}$, post-load glucose level $\geq 200$ $\mathrm{mg} / \mathrm{dL}, \mathrm{HbAlc} \geq 6.5 \%$, or use of insulin or oral antidiabetic drugs. Charlson Comorbidity Index was assessed using the method described previously [21]. The 10-year atherosclerotic cardiovascular disease (ASCVD) risk scores were calculated according to the risk assessment equation from the 2013 ACC/AHA guidelines [22]. After an overnight fast, venous samples were collected to determine hemoglobin, serum urea nitrogen, creatinine, fasting plasma glucose, albumin, calcium, phosphate, total cholesterol, triglycerides (TG), high-density lipoprotein cholesterol, LDL-C, and high-sensitivity C-reactive protein levels. Serum creatinine was measured by an isotope dilution mass spectrometry-traceable method at the central laboratory (Lab Genomics, Seoul, Korea). The CKD Epidemiology Collaboration equation was used to determine eGFR [23]. The second voided or random urine was collected, and samples were immediately sent to the central laboratory. Proteinuria was determined by 24-hour urinary protein excretion test (g/24 hr).

\section{Intensity of statin therapy}

Intensity of statin therapy was defined based on the expected LDL-C reduction indicated in clinical trials, as summarized in the 2013 ACC/AHA guidelines (Supplementary Table 1; available online) [14].

\section{Study outcomes}

Study participants were followed-up until March 31, 2018. The study endpoint was a composite of doubling of serum creatinine, $\geq 50 \%$ decline in eGFR, initiation of dialysis, or kidney transplantation.

\section{Statistical analysis}

All statistical analyses were performed using IBM SPSS software for Windows ver. 24.0 (IBM Corp., Armonk, NY, USA), SAS software ver. 9.2 (SAS Institute Inc., Cary, NC, USA), and R software ver. 3.3.1 (http://www.R-project. org). Continuous variables were expressed as mean \pm standard deviation or median and interquartile range. Categorical variables were expressed as frequency with percentage. All continuous variables were tested for normality before statistical analysis. The KolmogorovSmirnov test was performed to determine the normality of parameter distribution. Comparisons between groups were performed using analysis of variance with a normal distribution and the chi-square test or Fisher's exact test for categorical variables. Data that did not show a normal distribution were compared using the Kruskal-Wallis test. To evaluate the association between statin intensity and CKD progression, multivariable Cox regression analysis was performed. Variables that were significant in univariable analysis $(P<0.05)$ or clinically important were included for adjustment. The results were presented as hazard ratios (HRs) and 95\% confidence intervals (CIs). Participants who were lost to follow-up were censored at the date of the last examination. For all analyses, $P<0.05$ was considered significant.

\section{Results}

\section{Baseline characteristics}

The baseline characteristics of participants according to statin therapy intensity-based group are described in Table 1. Totals of 427 (39.8\%), 596 (55.5\%), and 50 (4.7\%) participants were classified into low-, moderate-, and high-intensity statin groups, respectively. The mean age of participants was $56.0 \pm 11.39$ years, and 665 (62.0\%) were male. The mean eGFR was $51.7 \pm 26.7 \mathrm{~mL} / \mathrm{min} / 1.73$ $\mathrm{m}^{2}$. Demographic data, including age, sex, smoking status, marital status, education status, income status, BMI, and $\mathrm{BP}$, showed no significant differences among the groups. The most common cause of CKD in study participants was glomerulonephritis, followed by diabetes and hypertension. However, no differences were found 
Table 1. Baseline characteristics of participants according to statin therapy intensity

\begin{tabular}{|c|c|c|c|c|c|}
\hline \multirow{2}{*}{ Characteristic } & \multirow{2}{*}{ Total $(n=1,073)$} & \multicolumn{4}{|c|}{ Statin therapy intensity group } \\
\hline & & Low $(n=427)$ & Moderate $(n=596)$ & High $(n=50)$ & $P$ value \\
\hline \multicolumn{6}{|l|}{ Demographic data } \\
\hline Age (yr) & $56.0 \pm 11.4$ & $56.3 \pm 11.1$ & $55.8 \pm 11.4$ & $55.2 \pm 13.4$ & 0.70 \\
\hline Male & $665(62.0)$ & $273(63.9)$ & $357(59.9)$ & $35(70.0)$ & 0.63 \\
\hline Smoker & & & & & 0.24 \\
\hline Never & $557(52.0)$ & $215(50.5)$ & $318(53.4)$ & $24(48.0)$ & \\
\hline Past & $179(16.7)$ & $64(15.0)$ & $103(17.3)$ & $12(24.0)$ & \\
\hline Current & $333(31.1)$ & $145(34.0)$ & $174(29.2)$ & $14(28.0)$ & \\
\hline Married & $916(85.7)$ & $364(85.4)$ & $511(86.2)$ & $41(82.0)$ & 0.91 \\
\hline Education & & & & & 0.73 \\
\hline Low & $274(25.7)$ & $97(22.8)$ & $167(28.2)$ & $10(20.0)$ & \\
\hline Intermediate & $378(35.4)$ & 169 (39.7) & $197(33.3)$ & $12(24.0)$ & \\
\hline High & $416(39.0)$ & $160(37.6)$ & $228(38.5)$ & $28(56.0)$ & \\
\hline Income & & & & & 0.49 \\
\hline High & $235(22.4)$ & $99(24.1)$ & $123(21.0)$ & $13(26.0)$ & \\
\hline Intermediate & $559(53.3)$ & $214(52.1)$ & $322(54.9)$ & $23(46.0)$ & \\
\hline Low & $254(24.2)$ & $98(23.8)$ & $142(24.4)$ & $14(28.0)$ & \\
\hline Body mass index (kg/m) & $25.2 \pm 5.7$ & $25.3 \pm 8.1$ & $25.0 \pm 3.2$ & $26.2 \pm 4.9$ & 0.28 \\
\hline Systolic BP (mmHg) & $128.7 \pm 15.9$ & $129.8 \pm 17.4$ & $127.7 \pm 15.0$ & $130.1 \pm 13.9$ & 0.11 \\
\hline Diastolic BP (mmHg) & $76.5 \pm 10.8$ & $77.1 \pm 11.8$ & $76.3 \pm 10.2$ & $74.5 \pm 10.5$ & 0.20 \\
\hline \multicolumn{6}{|l|}{ Comorbidities } \\
\hline Diabetes mellitus & $440(41.0)$ & $176(41.2)$ & $241(40.4)$ & $23(46.0)$ & 0.86 \\
\hline Hypertension & $1,059(98.7)$ & $424(99.3)$ & $586(98.3)$ & $49(98.0)$ & 0.17 \\
\hline Coronary artery disease & $97(9.0)$ & $42(9.8)$ & $48(8.1)$ & $7(14.0)$ & 0.89 \\
\hline Congestive heart failure & $24(2.2)$ & $7(1.6)$ & $15(2.5)$ & $2(4.0)$ & 0.34 \\
\hline Peripheral artery disease & $57(5.3)$ & $21(4.9)$ & $34(5.7)$ & $2(4.0)$ & 0.81 \\
\hline Charlson comorbidity index & $4.0(2.0-5.0)$ & $4.0(2.0-5.0)$ & $4.0(2.0-5.0)$ & $4.0(0.7-5.0)$ & 0.50 \\
\hline ASCVD risk score & $7.8(2.5-18.0)$ & $7.9(2.6-19.0)$ & $7.6(2.4-17.6)$ & $9.1(2.3-17.2)$ & 0.55 \\
\hline Cause of CKD & & & & & 0.82 \\
\hline Diabetic nephropathy & $291(27.1)$ & 119 (27.9) & $156(26.2)$ & $16(32.0)$ & \\
\hline Hypertension & $220(20.5)$ & $97(22.7)$ & $109(18.3)$ & $14(28.0)$ & \\
\hline Glomerulonephritis & $401(37.4)$ & 142 (33.3) & $242(40.6)$ & $17(34.0)$ & \\
\hline Polycystic kidney disease & $92(8.6)$ & $40(9.4)$ & $51(8.6)$ & $1(2.0)$ & \\
\hline Others & $69(6.4)$ & $29(6.8)$ & $38(6.4)$ & $2(4.0)$ & \\
\hline \multicolumn{6}{|l|}{ Use of other lipid-lowering agent } \\
\hline Ezetimibe & $118(11.0)$ & $79(18.5)$ & $36(6.0)$ & $3(6.0)$ & $<0.001$ \\
\hline Fibrate & $20(1.9)$ & $6(1.4)$ & $10(1.7)$ & $4(8.0)$ & 0.05 \\
\hline Nicotinic acid & $2(0.2)$ & $1(0.2)$ & $1(0.2)$ & $0(0.0)$ & 0.71 \\
\hline \multicolumn{6}{|l|}{ Laboratory parameters } \\
\hline eGFR (mL/min/1.73 m²) & $51.7 \pm 26.7$ & $51.3 \pm 26.2$ & $52.1 \pm 26.6$ & $51.5 \pm 32.0$ & 0.88 \\
\hline Proteinuria (g/24 hr) & $0.70(0.25-1.84)$ & $0.65(0.23-1.59)$ & $0.72(0.26-1.95)$ & $1.37(0.40-3.9)$ & 0.01 \\
\hline Hemoglobin (g/dL) & $13.2 \pm 7.1$ & $13.5 \pm 11.0$ & $12.9 \pm 1.9$ & $13.0 \pm 2.1$ & 0.33 \\
\hline Fasting plasma glucose (mg/dL) & $115.6 \pm 44.1$ & $117.3 \pm 41.2$ & $114.6 \pm 46.4$ & $113.4 \pm 40.2$ & 0.58 \\
\hline Albumin $(\mathrm{g} / \mathrm{dL})$ & $4.1 \pm 0.4$ & $4.2 \pm 0.4$ & $4.1 \pm 0.5$ & $3.9 \pm 0.6$ & 0.001 \\
\hline Calcium (mg/dL) & $9.1 \pm 0.5$ & $9.2 \pm 0.5$ & $9.1 \pm 0.5$ & $9.1 \pm 0.5$ & 0.45 \\
\hline Phosphate (mg/dL) & $3.7 \pm 0.7$ & $3.7 \pm 0.6$ & $3.7 \pm 0.6$ & $3.8 \pm 0.5$ & 0.54 \\
\hline Cholesterol (mg/dL) & $166.2 \pm 41.1$ & $167.1 \pm 38.8$ & $165.1 \pm 41.5$ & $171.4 \pm 53.9$ & 0.48 \\
\hline Triglycerides (mg/dL) & $165.2 \pm 102.5$ & $160.9 \pm 97.2$ & $165.1 \pm 103.7$ & $203.7 \pm 126.1$ & 0.02 \\
\hline $\mathrm{HDL}-\mathrm{C}(\mathrm{mg} / \mathrm{dL})$ & $49.0 \pm 14.9$ & $49.3 \pm 15.4$ & $48.8 \pm 14.6$ & $48.8 \pm 14.2$ & 0.85 \\
\hline LDL-C (mg/dL) & $89.0 \pm 32.1$ & $90.5 \pm 30.2$ & $88.0 \pm 32.3$ & $89.1 \pm 44.1$ & 0.48 \\
\hline $\mathrm{hs}-\mathrm{CRP}(\mathrm{mg} / \mathrm{dL})$ & $0.60(0.26-1.60)$ & $0.60(0.29-1.60)$ & $0.60(0.20-1.70)$ & $0.69(0.40-1.25)$ & 0.90 \\
\hline
\end{tabular}

Data are presented as mean \pm standard deviation, number (\%), or median (interquartile range).

ASCVD, atherosclerotic cardiovascular disease; BP, blood pressure; CKD, chronic renal disease; eGFR, estimated glomerular filtration rate; HDL-C, high-density lipoprotein cholesterol; LDL-C, low-density lipoprotein cholesterol; hs-CRP, high-sensitivity C-reactive protein. 
in cause of CKD among the three groups. Regarding use of other lipid-lowering drugs, the high-intensity group was taking less ezetimibe and more fibrates than the lowintensity group. In the laboratory data, the high-intensity group showed higher level of proteinuria and lower level of serum albumin than the low-intensity group. There were no differences in lipid profiles among groups except in terms of triglyceride level; patients in the highintensity group showed higher level of TG than those in the low-intensity group. The ASCVD risk score, Charlson Comorbidity Index, and prevalence of comorbidities such as diabetes, hypertension, coronary artery disease, congestive heart failure, and peripheral artery disease were similar among groups.

\section{Risk of kidney disease progression according to intensity of statin therapy}

During a median follow-up of 39.9 (25.4-61.6) months,

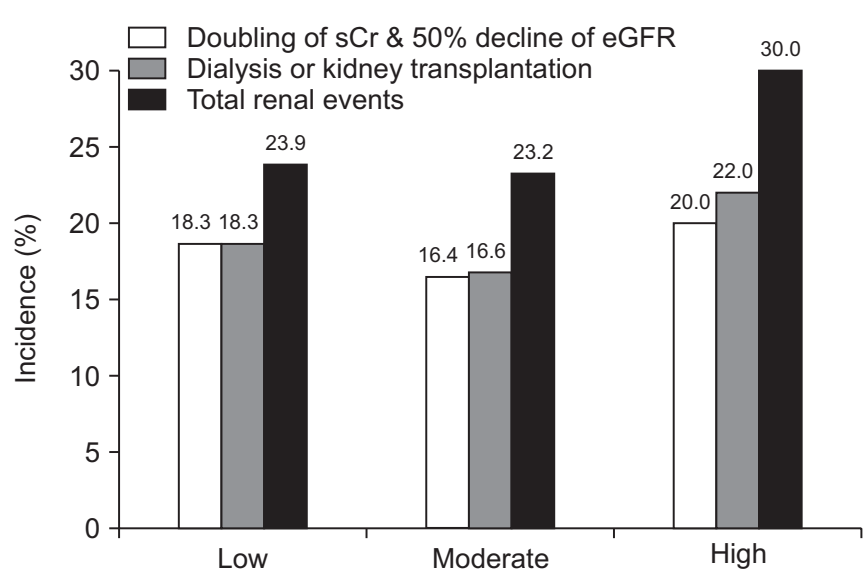

Figure 2. Incidence of renal events across statin intensity groups. eGFR, estimated glomerular filtration rate; $\mathrm{sCr}$, serum creatinine.
$102(23.9 \%), 138(23.2 \%)$, and $15(30.0 \%)$ renal outcome events occurred in low-, moderate-, and high-intensity groups $(P$ for trend $=0.74$ ), respectively. The crude incidence rate of doubling of serum creatinine, $\geq 50 \%$ decline in eGFR, and initiation of dialysis or kidney transplantation was higher in the high-intensity group than in the low-intensity group without statistical significance (Fig. 2). However, Kaplan-Meier analysis showed no significant difference in time to first adverse kidney outcome among statin intensity groups (Fig. 3). In multivariable Cox analysis adjusted for demographic factors of age, sex, BMI, SBP, smoking status, income status, comorbidities (hypertension, diabetes, and CVDs), laboratory data (eGFR, proteinuria, and lipid profiles), and use of other lipid-lowering agents (ezetimibe or fibrates), use of highor moderate-intensity statin was not associated with risk of CKD progression compared with use of low-intensity statin. The corresponding HRs (95\% CIs) were 1.15 (0.60$2.20)$ and $0.97(0.72-1.30)$, respectively (Table 2$)$. In ad-

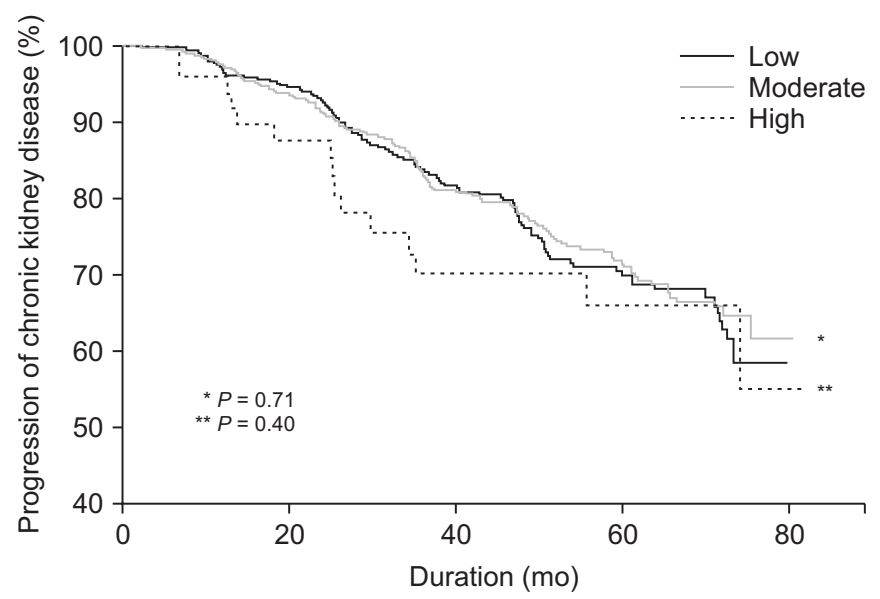

Figure 3. Kaplan-Meier curve for progression of chronic kidney disease according to statin therapy intensity.

Table 2. Association between progression of chronic kidney disease and statin therapy intensity

\begin{tabular}{|c|c|c|c|c|c|c|c|c|c|}
\hline & \multicolumn{3}{|c|}{ Model 1} & \multicolumn{2}{|l|}{ Model 2} & \multicolumn{2}{|l|}{ Model 3} & \multicolumn{2}{|l|}{ Model 4} \\
\hline & Cases $(\%)^{a}$ & $\mathrm{HR}(95 \% \mathrm{Cl})$ & $P$ & $\mathrm{HR}(95 \% \mathrm{Cl})$ & $P$ & $\mathrm{HR}(95 \% \mathrm{Cl})$ & $P$ & $\mathrm{HR}(95 \% \mathrm{Cl})$ & $P$ \\
\hline Low & $102(23.9)$ & \multicolumn{8}{|c|}{ Reference } \\
\hline Moderate & $138(23.2)$ & $0.95(0.74-1.23)$ & 0.71 & $1.00(0.77-1.29)$ & 0.99 & $0.94(0.73-1.23)$ & 0.66 & $0.97(0.72-1.30)$ & 0.82 \\
\hline High & $15(30.0)$ & $1.26(0.73-2.17)$ & 0.40 & $1.29(0.75-2.23)$ & 0.36 & $1.34(0.77-2.34)$ & 0.30 & $1.15(0.60-2.20)$ & 0.69 \\
\hline
\end{tabular}

$\mathrm{Cl}$, confidence interval; $\mathrm{HR}$, hazard ratio.

Model 1: unadjusted; Model 2: adjusted for age, sex, body mass index, and systolic blood pressure; Model 3: adjusted for Model $2+$ smoking, income status, comorbidities (histories of hypertension, diabetes, and cardiovascular disease); Model 4: adjusted for Model 3 + laboratory factors (estimated glomerular filtration rate; proteinuria; lipid profiles including triglycerides, high-density lipoprotein cholesterol, and low-density lipoprotein cholesterol) and use of other lipid-lowering agents (ezetimibe or fibrate).

${ }^{\mathrm{a}} \mathrm{P}$ for trend $=0.74$. 


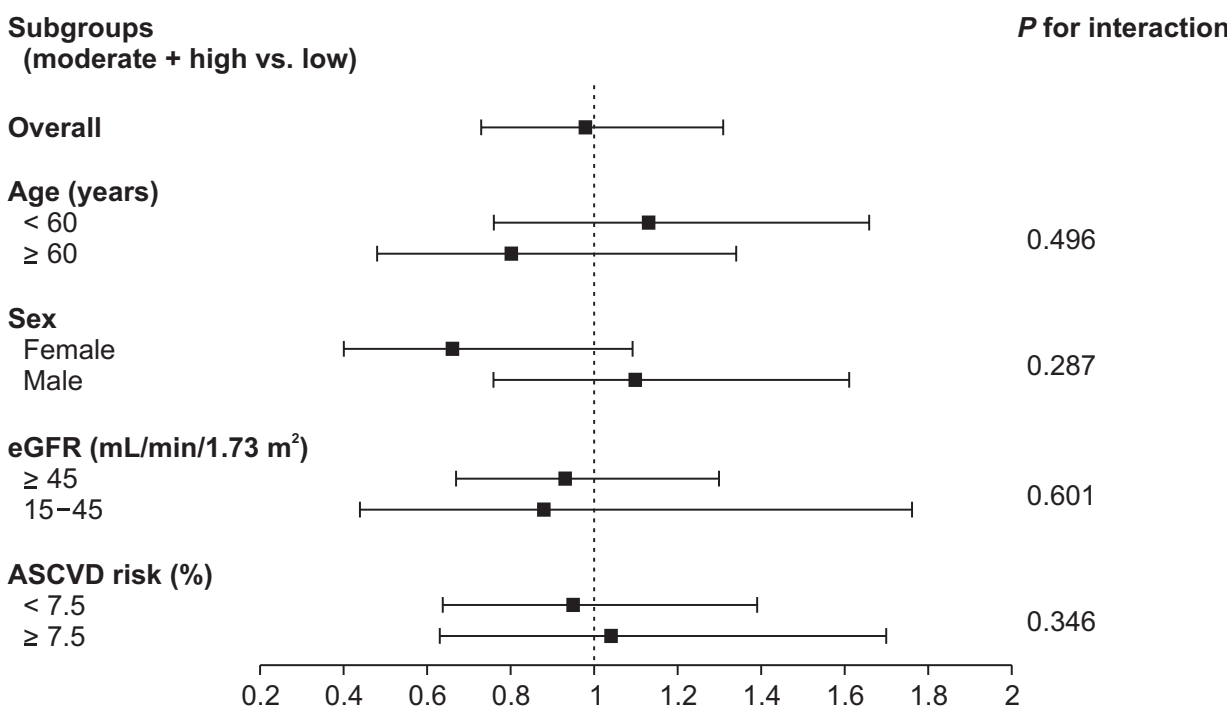

Figure 4. Subgroup analysis. ASCVD, atherosclerotic cardiovascular disease; eGFR, estimated glomerular filtration rate. dition, the annual eGFR decline rate was not different among the three groups $(-1.19 \pm 0.24,-1.58 \pm 0.16$, and $-0.16 \pm 0.59 \mathrm{~mL} / \mathrm{min} / 1.73 \mathrm{~m}^{2} / \mathrm{yr}$ in low-, moderate-, and high-intensity statin groups, respectively; $P=0.06$ ). When high- and moderate-intensity statins were grouped together, no significant association was observed between statin intensity and primary outcome (HR, 1.03; 95\% CI, 0.77-1.38; Supplementary Table 2).

In addition, the association between statin intensity and change in proteinuria during follow-up was evaluated. Proteinuria level was not significantly different among statin intensity groups during the follow-up pe$\operatorname{riod}(P=0.64 ;$ Supplementary Fig. 1$)$.

\section{Subgroup analyses}

We further evaluated the association between statin therapy intensity and CKD progression in subgroups stratified by age ( $<60$ years vs. $\geq 60$ years), sex (female vs. male), eGFR category ( $15-45$ vs. $\geq 45 \mathrm{~mL} / \mathrm{min} / 1.73 \mathrm{~m}^{2}$ ), and ASCVD risk score ( $<7.5 \%$ vs. $\geq 7.5 \%)$. There were no significant interactions between stratified variables and statin therapy intensity group in terms of CKD progression. This result suggests that the association was consistent regardless of age, sex, eGFR, and ASCVD risk score (Fig. 4).

\section{Discussion}

In the present study, we investigated the association between statin therapy intensity and CKD progression in patients with CKD. Despite the crucial role of intensitybased statin therapy in preventing cardiovascular events, low-, moderate-, or high-intensity statin therapies were similarly associated with risk of adverse kidney outcome. This association was consistent regardless of age, sex, eGFR category, and ASCVD risk score.

Dyslipidemia is an important risk factor for adverse cardiovascular outcome in patients with and without CKD [24]. Previous meta-analyses have shown that statin therapies largely contribute to reduced atherosclerotic cardiovascular events and related mortality in patients with non-dialysis-dependent CKD [25,26]. In addition, dyslipidemia is associated with more rapid loss of kidney function in people without kidney disease. Patients with kidney disease are more likely to have hyperlipidemia, although not all patients with elevated lipid levels have kidney disease $[27,28]$. In experimental studies, hyperlipidemia may exacerbate preexisting kidney disease by causing direct injury to the glomerular basement membrane [29]. In contrast, statins may improve kidney function by improving endothelial function to increase renal perfusion [30]. Furthermore, statins reduce proteinuria by attenuating renal inflammation and fibrosis in the tubulointerstitium [31]. Although lipid accumulation and lipotoxicity may lead to kidney injury in experimental animal models, there is insufficient evidence supporting an effect of lipid profile abnormality on kidney function in humans [32]. Furthermore, epidemiologic studies have yielded conflicting results on the relationship between statins and kidney disease progression among patients with CKD. Several trials have suggested the beneficial ef- 
fects of statins on kidney disease outcomes [33,34]. However, previous studies were based on post hoc analyses of cardiovascular benefits of statin therapy, and the effects on kidney function were modest. Moreover, studies suggesting the reno-protective effects of statins mainly included patients with prevalent CVD, and several other studies showed that statins have no effect on kidney function in CKD patients without CVD $[35,36]$. Therefore, statin therapy may decrease the rate of kidney function decline mainly in patients with prevalent CVD. However, the clinical significance of this effect remains unclear.

Recently, statin therapies of differing intensity are being recommended. The ACC/AHA guidelines emphasize different statin therapy intensities in patients with varying risks of ASCVD [14]. In this regard, the dose-dependent relationship between statin therapy intensity and renal outcome can be hypothesized [37]. Sanguankeo et al [12] reported that high-intensity statin therapy improved eGFR in patients with CKD compared to a control group, but moderate- to low-intensity statin therapies were not associated with improved eGFR. They also showed that LDL-C reduction had a significant association with changes in eGFR; the magnitude of eGFR increase was well correlated with decrease in LDL-C concentration. In line with our study, however, they showed no significant association between statin therapy intensity and hard study endpoints, such as $50 \%$ eGFR reduction or development of ESRD. Possible reasons for the nonsignificant association between statin therapy intensity and hard renal events in this study are suggested. First, most previous studies that reported the effects of statins on renal function were post-hoc analyses of original studies that aimed to examine the cardiovascular benefits of statin therapy. Thus, almost all study subjects had preexisting CVD. However, in the present study, only $10 \%$ of study participants had previous CVD at baseline. It is possible that the protective effect of statins might not have been pronounced in our study cohort. Hence, subgroup analysis stratified by low vs. high ASCVD risk scores was performed; however, there was still no significant relationship between statin therapy intensity and renal event. Second, the positive effect of statins on declining kidney function may be attenuated in the presence of other risk factors for CKD progression, such as high BP. Hypertension is one of the strongest risk factors for kidney function decline. A previous study showed that the benefi- cial effect of statins on preservation of kidney function was weaker in people with higher SBP [38]. This result suggests that other risk factors can modify the effect of statins on kidney function. Third, previous studies indicated that statin therapy may lead to unintended acute kidney injury. In particular, use of high-intensity statin therapy was associated with an increased rate of acute kidney injury diagnosis during hospital admissions [18]. Therefore, the possible harmful effect of statin therapy might have hindered the reno-protective effect of statins. Nevertheless, statins also may have pleiotropic effects favorable for reducing CKD progression, such as lowering oxidative stress, reducing inflammation, and stabilizing atherosclerotic plaques [39]. Thus, further studies are warranted to validate the effect of statins on renal outcomes.

Although we did not observe significant association between statin intensity and adverse kidney outcome, our findings remain valuable. First, it is well-known that statin therapy in patients with CKD is not as effective as in people without CKD. The reason for this is unclear, but we thought that statin intensity might modify the relationship between statin use and adverse clinical outcome in patients with CKD. This prompted us to analyze this association in depth using a cohort database involving only patients with CKD. Although we could not assert a causal relationship, our findings suggest that the lower effectiveness of statin therapy in patients with CKD may not be related to statin intensity. Second, there is concern regarding the possible harmful association of highintensity statins with acute kidney injury [40]. However, our study showed that high- or low-intensity statin use was similarly associated with risk of CKD progression. This indicates that long-term kidney outcomes may not be affected by statin intensity.

This study has several limitations. First, due to its observational design, the causal relationship between statin therapy intensity and kidney function could not be determined. Second, patients with ESRD requiring dialysis or those who had received kidney transplantation were not included. Thus, the study results cannot be generalized to these patients. Third, only baseline statin therapy was used in the analysis; thus, the study results cannot reflect changes in statin use over time. Furthermore, changes in LDL-C level according to statin intensity could not be evaluated because patients had been treated with statin 
therapy before study enrollment. The beneficial effects of high-intensity statin therapy were linked to higher reduction in LDL-C level [17]. Thus, it could not be determined whether high-intensity statin therapy with a large reduction in LDL-C level has a reno-protective effect. Fourth, the proportion of participants in the high-intensity statin therapy group was relatively small. The 2013 KDIGO lipid guidelines recommend statins for patients aged $\geq$ 50 years with CKD and with $\mathrm{eGFR}<60 \mathrm{~mL} / \mathrm{min} / 1.73 \mathrm{~m}^{2}$, but it does not specify statin intensity dosing recommendations [41]. Thus, most patients with CKD take statins irrespective of dose based on statin intensity. In the present study, ASCVD risk scores were not significantly different among statin therapy intensity groups, suggesting that statins were not prescribed based on intensity-based dosing recommendations. This may have resulted in a decreased effect of statins on kidney function. Finally, the study cohort included a single ethnic group, with all participants being Korean. Thus, the results should be interpreted with caution, and our findings may not be generalized to other ethnic groups.

In conclusion, among Korean adults with CKD, we did not observe any significant association between statin therapy intensity and CKD progression. However, the findings obtained from our observational study do not provide sufficient evidence on this issue. Given the importance of treating dyslipidemia in patients with CKD, further large and well-designed randomized controlled trials are needed to clearly determine the effects of intensity-based statin therapy on kidney function.

\section{Conflicts of interest}

All authors have no conflicts of interest to declare.

\section{Acknowledgments}

The authors acknowledge all patients who participated in the KNOW-CKD and the investigators who collected data. This work was supported by a research program funded by the Korea Centers for Disease Control and Prevention (2011E3300300, 2012E3301100, 2013E3301600, 2013E3301601, 2013E3301602, and 2016E3300200). The funding sources had no role in the design or conduct of the study; collection, management, analysis, or interpretation of the data; preparation, review, or approval of the manuscript; or the decision to submit the manuscript for publication.

\section{Authors' contributions}

Jong Hyun Jhee participated in creating research idea, study design, acquisition, analysis and interpretation of the data and wrote the manuscript. Seung Hyeok Han participated in creating research idea, study design, data analysis/interpretation, supervision of the study and wrote the manuscript. Young Su Joo participated in data acquisition and analysis and helped to draft the maunscript. Jung Tak Park, Tae-Hyun Yoo, Sue Kyung Park, Ji Yong Jung, Soo Wan Kim, Yun Kyu Oh, Kook-Hwan Oh, Shin-Wook Kang, Kyu Hun Choi, and Curie Ahn provided intellectual content of critical importance to the work and helped to draft the manuscript. All authors read and approved the final manuscript.

\section{References}

[1] US Renal Data System 2019 annual data report: epidemiology of kidney disease in the United States. Am J Kidney Dis 2019 Oct 31 [Epub]. Doi: 10.1053/j.ajkd.2019.09.002.

[2] Kim KM, Oh HJ, Choi HY, Lee H, Ryu DR. Impact of chronic kidney disease on mortality: a nationwide cohort study. Kidney Res Clin Pract 2019;38:382-390.

[3] Chronic Kidney Disease Prognosis Consortium, Matsushita $\mathrm{K}$, van der Velde $\mathrm{M}$, et al. Association of estimated glomerular filtration rate and albuminuria with all-cause and cardiovascular mortality in general population cohorts: a collaborative meta-analysis. Lancet 2010;375:2073-2081.

[4] Astor BC, Matsushita K, Gansevoort RT, et al. Lower estimated glomerular filtration rate and higher albuminuria are associated with mortality and end-stage renal disease. A collaborative meta-analysis of kidney disease population cohorts. Kidney Int 2011;79:1331-1340.

[5] Foley RN, Parfrey PS, Sarnak MJ. Clinical epidemiology of cardiovascular disease in chronic renal disease. Am J Kidney Dis 1998;32(5 Suppl 3):S112-S119.

[6] Mann JF, Gerstein HC, Pogue J, Bosch J, Yusuf S. Renal insufficiency as a predictor of cardiovascular outcomes and the impact of ramipril: the HOPE randomized trial. Ann Intern Med 2001;134:629-636.

[7] Lee C, Yun HR, Joo YS, et al. Framingham risk score and risk of incident chronic kidney disease: a community-based 
prospective cohort study. Kidney Res Clin Pract 2019;38: 49-59.

[8] Parikh NI, Hwang SJ, Larson MG, Meigs JB, Levy D, Fox CS. Cardiovascular disease risk factors in chronic kidney disease: overall burden and rates of treatment and control. Arch Intern Med 2006;166:1884-1891.

[9] Bussolati B, Deregibus MC, Fonsato V, et al. Statins prevent oxidized LDL-induced injury of glomerular podocytes by activating the phosphatidylinositol 3-kinase/AKT-signaling pathway. J Am Soc Nephrol 2005;16:1936-1947.

[10] Guijarro C, Egido J. Transcription factor-kappa B (NF-kappa B) and renal disease. Kidney Int 2001;59:415-424.

[11] Nam KH, Chang TI, Joo YS, et al.; KNOW-CKD (KoreaN Cohort Study for Outcomes in Patients With Chronic Kidney Disease) Investigators. Association between serum highdensity lipoprotein cholesterol levels and progression of chronic kidney disease: results from the KNOW-CKD. J Am Heart Assoc 2019;8:e011162.

[12] Sanguankeo A, Upala S, Cheungpasitporn W, Ungprasert $P$, Knight EL. Effects of statins on renal outcome in chronic kidney disease patients: a systematic review and metaanalysis. PLoS One 2015;10:e132970.

[13] Su X, Zhang L, Lv J, et al. Effect of statins on kidney disease outcomes: a systematic review and meta-analysis. Am J Kidney Dis 2016;67:881-892.

[14] Stone NJ, Robinson JG, Lichtenstein AH, et al.; American College of Cardiology/American Heart Association Task Force on Practice Guidelines. 2013 ACC/AHA guideline on the treatment of blood cholesterol to reduce atherosclerotic cardiovascular risk in adults: a report of the American College of Cardiology/American Heart Association Task Force on Practice Guidelines. J Am Coll Cardiol 2014;63:28892934.

[15] Rodriguez F, Maron DJ, Knowles JW, Virani SS, Lin S, Heidenreich PA. Association between intensity of statin therapy and mortality in patients with atherosclerotic cardiovascular disease. JAMA Cardiol 2017;2:47-54.

[16] Wanner C, Tonelli M; Kidney Disease: Improving Global Outcomes Lipid Guideline Development Work Group Members. KDIGO Clinical Practice Guideline for Lipid Management in CKD: summary of recommendation statements and clinical approach to the patient. Kidney Int 2014;85:1303-1309.

[17] Baigent C, Landray MJ, Reith C, et al.; SHARP Investigators. The effects of lowering LDL cholesterol with simvastatin plus ezetimibe in patients with chronic kidney disease (study of heart and renal protection): a randomised placebo-controlled trial. Lancet 2011;377:2181-2192.

[18] Dormuth CR, Hemmelgarn BR, Paterson JM, et al.; Canadian Network for Observational Drug Effect Studies. Use of high potency statins and rates of admission for acute kidney injury: multicenter, retrospective observational analysis of administrative databases. BMJ 2013;346:f880.

[19] Corrao G, Soranna D, Casula M, Merlino L, Porcellini MG, Catapano AL. High-potency statins increase the risk of acute kidney injury: evidence from a large populationbased study. Atherosclerosis 2014;234:224-229.

[20] Oh KH, Park SK, Park HC, et al.; Representing KNOW-CKD Study Group. KNOW-CKD (KoreaN cohort study for Outcome in patients With Chronic Kidney Disease): design and methods. BMC Nephrol 2014;15:80.

[21] Charlson ME, Pompei P, Ales KL, MacKenzie CR. A new method of classifying prognostic comorbidity in longitudinal studies: development and validation. J Chronic Dis 1987;40:373-383.

[22] Goff DC Jr, Lloyd-Jones DM, Bennett G, et al. 2013 ACC/ AHA guideline on the assessment of cardiovascular risk: a report of the American College of Cardiology/American Heart Association Task Force on Practice Guidelines. J Am Coll Cardiol 2014;63(25 Pt B):2935-2959.

[23] Levey AS, Stevens LA, Schmid CH, et al.; CKD-EPI (Chronic Kidney Disease Epidemiology Collaboration). A new equation to estimate glomerular filtration rate. Ann Intern Med 2009;150:604-612.

[24] Herzog CA, Asinger RW, Berger AK, et al. Cardiovascular disease in chronic kidney disease. A clinical update from Kidney Disease: Improving Global Outcomes (KDIGO). Kidney Int 2011;80:572-586.

[25] Palmer SC, Craig JC, Navaneethan SD, Tonelli M, Pellegrini F, Strippoli GF. Benefits and harms of statin therapy for persons with chronic kidney disease: a systematic review and meta-analysis. Ann Intern Med 2012;157:263-275.

[26] Major RW, Cheung CK, Gray LJ, Brunskill NJ. Statins and Cardiovascular Primary Prevention in CKD: A Meta-Analysis. Clin J Am Soc Nephrol 2015;10:732-739.

[27] Schaeffner ES, Kurth T, Curhan GC, et al. Cholesterol and the risk of renal dysfunction in apparently healthy men. $J$ Am Soc Nephrol 2003;14:2084-2091.

[28] Muntner P, Coresh J, Smith JC, Eckfeldt J, Klag MJ. Plasma lipids and risk of developing renal dysfunction: the atherosclerosis risk in communities study. Kidney Int 2000;58: 293-301. 
[29] Moorhead JF. Lipids and progressive kidney disease. Kidney Int Suppl 1991;31:S35-S40.

[30] O'Driscoll G, Green D, Taylor RR. Simvastatin, an HMGcoenzyme A reductase inhibitor, improves endothelial function within 1 month. Circulation 1997;95:1126-1131.

[31] Zoja C, Corna D, Camozzi D, et al. How to fully protect the kidney in a severe model of progressive nephropathy: a multidrug approach. J Am Soc Nephrol 2002;13:2898-2908.

[32] Bobulescu IA. Renal lipid metabolism and lipotoxicity. Curr Opin Nephrol Hypertens 2010;19:393-402.

[33] Huskey J, Lindenfeld J, Cook T, et al. Effect of simvastatin on kidney function loss in patients with coronary heart disease: findings from the Scandinavian Simvastatin Survival Study (4S). Atherosclerosis 2009;205:202-206.

[34] Tonelli M, Isles C, Craven T, et al. Effect of pravastatin on rate of kidney function loss in people with or at risk for coronary disease. Circulation 2005;112:171-178.

[35] Kendrick J, Shlipak MG, Targher G, Cook T, Lindenfeld J, Chonchol M. Effect of lovastatin on primary prevention of cardiovascular events in mild CKD and kidney function loss: a post hoc analysis of the Air Force/Texas Coronary Atherosclerosis Prevention Study. Am J Kidney Dis 2010;55: 42-49.
[36] Haynes R, Lewis D, Emberson J, et al.; SHARP Collaborative Group; SHARP Collaborative Group. Effects of lowering LDL cholesterol on progression of kidney disease. J Am Soc Nephrol 2014;25:1825-1833.

[37] Vogt L, Bangalore S, Fayyad R, et al. Atorvastatin has a dose-dependent beneficial effect on kidney function and associated cardiovascular outcomes: post hoc analysis of 6 double-blind randomized controlled trials. J Am Heart Assoc 2019;8:e010827.

[38] Esmeijer K, Dekkers OM, de Fijter JW, Dekker FW, Hoogeveen EK. Effect of different types of statins on kidney function decline and proteinuria: a network meta-analysis. Sci Rep 2019;9:16632.

[39] Zhou Q, Liao JK. Pleiotropic effects of statins: basic research and clinical perspectives. Circ J2010;74:818-826.

[40] Tonelli M, Lloyd AM, Bello AK, et al.; Alberta Kidney Disease Network. Statin use and the risk of acute kidney injury in older adults. BMC Nephrol 2019;20:103.

[41] Walther CP, Richardson PA, Virani SS, Winkelmayer WC, Navaneethan SD. Association between intensity of statin therapy and mortality in persons with chronic kidney disease. Nephrol Dial Transplant 2020;35:312-319. 